\title{
Metallization of Silicon upon Potassium Adsorption
}

\author{
S. Ciraci ${ }^{(a)}$ and Inder P. Batra ${ }^{(b)}$ \\ IBM Zurich Research Laboratory, 8803 Rüschlikon, Switzerland \\ (Received 24 September 1986)
}

\begin{abstract}
We report novel features of potassium deposition on a $\mathrm{Si}(111)-(2 \times 1)$ surface as a function of coverage. The binding is ionic even at the saturation coverage without any overlayer metallization. Up to a threshold coverage, the alkali-metal electrons are donated to the empty surface state resulting in a 1D metallic chain. Above this coverage, the conduction-band states are occupied, so that the surface electrons become itinerant leading to the metallization of the substrate and onset of enhanced conductivity.

PACS numbers: $71.30 .+\mathrm{h}, 71.45 . \mathrm{Gm}, 73.40 . \mathrm{Ns}$
\end{abstract}

Generation of desired electronic properties by metal deposition on the semiconductor surfaces has been exploited in a number of technical applications in the area of microelectronics. It is also now quite evident that one might expect interesting coverage-dependent effects, and, in particular, the overlayer metallization may set in at a critical threshold. For those semiconductors which have active surface states, the metallization of the overlayer must compete with the formation of metal-semiconductor bonds. In fact, depending on the relative value of the metallic cohesive energy and the metal-substrate interaction energy the overlayer metallization may be suppressed altogether.

In this Letter, we report novel features of $\mathrm{Si}(111)$ $(2 \times 1)$ substrate metallization upon deposition of potassium as a function of coverage. Our calculations show that, up to a threshold coverage, the alkali-metal electrons are donated to the empty $\pi^{*}$ band ${ }^{1}$ resulting in a $1 \mathrm{D}$ metallic system. Above this coverage, the Fermi level crosses the bulk conduction-band minimum, so that the surface electrons become itinerant leading to the metallization of the substrate. An important aspect of this prediction is that the excess carrier concentration in the conduction band can be controlled at will by $\mathrm{K}$ deposition. The critical adsorption coverage gives rise to the same physical effects as bulk doping would, but without the introduction of any scattering centers in the bulk (and hence high mobility may be expected). We also show that the $\pi$-bonded chain structure ${ }^{1}$ is stable under $\mathrm{K}$ adsorption. These conclusions are derived from totalenergy minimization, force, and electronic-structure calculations on the monolayer, multilayer, and bulk potassi$\mathrm{um}$, as well as the clean and K-covered $\mathrm{Si}$ surfaces.

Three key ingredients responsible for the unusual interaction between $\mathrm{K}$ and the $\mathrm{Si}(111)$ surface are (i) a saturation coverage above the monolayer, (ii) ionic binding of the adsorbate even at saturation, and (iii) an empty surface-state band overlapping with the conductionband continua. An interesting feature of the $\mathrm{Si}(111)$ $(2 \times 1)$ surface is that the dangling orbitals on the chain atoms are closely spaced and hence lead to a large dispersion $(\sim 2 \mathrm{eV})$ of the surface-state bands. ${ }^{1}$ The bonding $\pi$ band contributes to the chain formation, and the $\pi^{*}$ band has an overlap with the conduction band in a sizable region of the surface Brillouin zone (BZ). Moreover, the topology of the surface charge density with two hole sites in the superlattice cell offers the possibility of accommodating alkali-metal atoms even beyond the monolayer coverage.

We used a repeating slab geometry for the selfconsistent pseudopotential ${ }^{2}$ calculations with the Bloch functions expanded in terms of $\sim 1100$ plane waves ( 600 treated exactly). We simulated the substrate by a slab consisting of six layers having the top surface in the $\pi$-chain configuration, and the back surface in ideal configuration, but saturated with hydrogen atoms. The adsorption of alkali-metal atoms is studied starting from one $\mathrm{K}(\Theta=1)$ up to three $\mathrm{K}$ atoms $(\Theta=3)$ in the surface unit cell. To find the equilibrium configuration, the total energies and forces for several possible adsorption sites of the $\mathrm{K}$ atoms (above the $\pi$ chain, above the second layer chain, and the tilted hexagon formed by surface and subsurface $\mathrm{Si}$ atoms) were calculated by our varying the adatom-surface height $h$. The potassium atoms lying 2.8 a.u. above the center of the tilted hexagon $(h=1.8$ a.u.) have the minimum total energy. This position coincides with one of the two hole sites of the surface charge density. The average $\mathrm{K}-\mathrm{Si}$ interatomic distance being only 5 a.u. is 1.6 a.u. shorter than the sum of atomic radii of $\mathrm{Si}$ and $\mathrm{K}$. Subtracting the atomic radius of $\mathrm{Si}$ from the calculated $\mathrm{K}-\mathrm{Si}$ distance, we obtained a radius of 2.7 a.u. for the adsorbed $\mathrm{K}$ which is very close to its ionic radius with inert-gas configuration. By use of the calculated total energies of the bulk and monolayer $\mathrm{K}$ arranged in the same slab unit cell, and those of the clean and $\mathrm{K}$-covered substrate, we determine the (adsorption) binding energy of the single $\mathrm{K}$ atom to be $2.8 \mathrm{eV}$.

For higher adsorbate coverage $(\Theta=2)$, the calculations are repeated by placement of an additional $\mathrm{K}$ atom at the second-hole site of the surface charge, and the binding energy is found to be $2.1 \mathrm{eV}$ (or an average binding energy of $2.5 \mathrm{eV}$ per $\mathrm{K}$ ). Because of the core repulsion between $\mathrm{K}$ atoms, the average binding energy for $\Theta=2$ is reduced. However, it is significantly large 
and indicates a strong interaction between $\mathrm{K}$ and $\mathrm{Si}$ substrates. For both $\Theta=1$ and $\Theta=2$, the calculated forces on the atoms, specifically forces on the chain atoms, are found to be small, presenting strong evidence that the chain structure is stable. Interestingly, the directions of these small forces indicate that as $\Theta$ increases, the chain atoms rise slightly and the $\mathrm{K}$ atoms are pulled downwards, so that $h$ becomes smaller. To simulate the higher coverage $\Theta=3$, the third $K$ atom is placed 9.7 a.u. above the surface. In this configuration, the interatomic distance with the lower-lying $\mathrm{K}$ atoms is set close to that of the bulk metal to allow for a metallic-bond formation. The comparison of total energies, however, shows that neither the third $\mathrm{K}$ atom is bound nor a metallic bond formed among the alkali-metal overlayer atoms. Metallization of the adsorbed $\mathrm{K}$ overlayer does not take place because it would cause $\mathrm{K}$ atoms to relax by regaining their electrons, so that they would lose $2.5 \mathrm{eV}$ per atom but gain only $1 \mathrm{eV}$ through metallic cohesion. On the basis of these energetic considerations, we conclude that up to $\Theta=2$ the binding is ionic. Analysis of the energy-band structure and the charge density provides further support for this conclusion.

Figure 1 illustrates calculated energy-band structures of the unsupported $\mathrm{K}$ monolayer, clean, and $\mathrm{K}$-covered Si surface. Three lowest $s$ and $p$ bands of the $\mathrm{K}$ monolayer have a large dispersion along the [110] direction. Because of the quantum size effect, ${ }^{3}$ the calculated work function is somewhat larger than that of the bulk metal. The form of the surface-state bands of the clean Si(111)- $(2 \times 1)$ are now well known. ${ }^{1,4}$ At the monolayer coverage, the Fermi level rises by $0.7 \mathrm{eV}$ with respect to the valence-band edge, and the $\pi^{*}$ band becomes partially occupied. This shows that the alkalimetal electrons are donated to the empty surface-state band. As seen, the overall form of the $\pi$ and $\pi^{*}$ bands (identified by an extensive charge-density analysis over the surface BZ for clean and K-covered substrate) stays nearly intact upon $\mathrm{K}$ adsorption, but the substrate surface undergoes a transition from an insulating to a metallic state. The calculated reduction of the work function from 4.5 to $2.9 \mathrm{eV}$ is consistent with the ionic picture proposed here. Above the threshold coverage (estimated to be $\Theta=0.9$ ) the extra charge is accommodated by the bulk conduction band, and the Fermi level stays almost pinned. (At $\Theta=2, E_{\mathrm{F}}$ moves up only $0.1 \mathrm{eV}$, and the work function decreases $<0.1 \mathrm{eV}$ relative to the $\Theta=1$ value.) Only at this high coverage stage do we observe a modification of the states above $E_{\mathrm{F}}$ (shown by dotted lines) to have an admixture from $\mathrm{K}-4 s$ orbitals. However, the lowest bands of the $\mathrm{K}$ layers are discarded upon adsorption.

Experimentally, the adsorption of $\mathrm{Cs}$ on $\mathrm{Si}(111)$ $(2 \times 1)$ has been studied. ${ }^{5}$ Since $\mathrm{K}$ and Cs have similar electronic properties, it is reasonable to compare our results at least qualitatively with $\mathrm{Cs}-\mathrm{Si}$ experimental data.
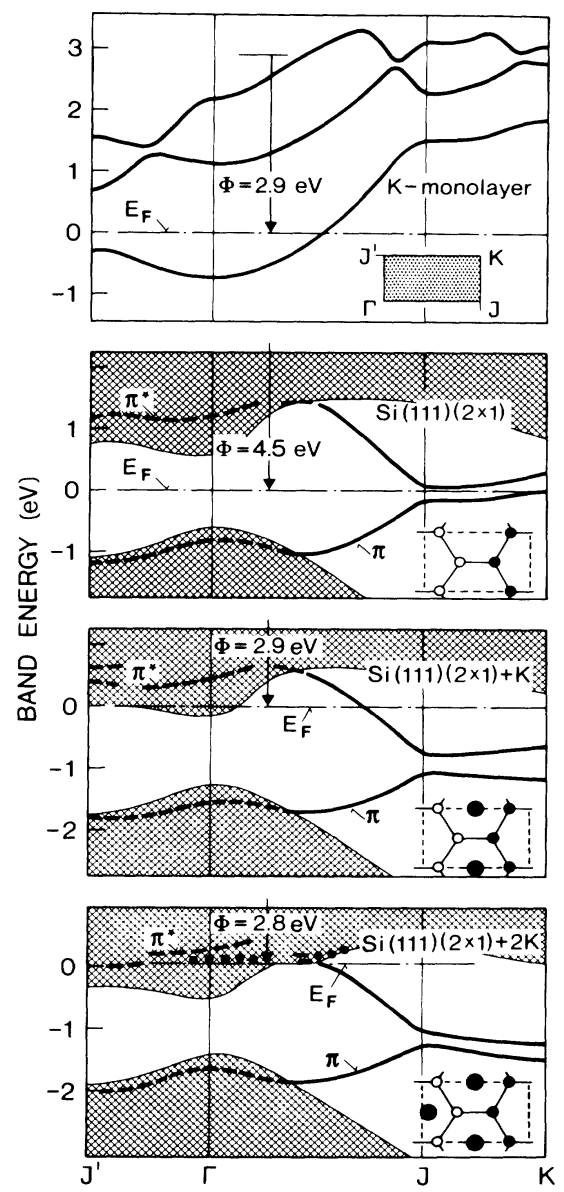

FIG. 1. Surface energy-band structure and the work function $(\Phi)$ of the unsupported $\mathrm{K}$ monolayer, and clean, as well as one-K- and two-K-adsorbed $\mathrm{Si}(111)-(2 \times 1)$ surfaces. The surface $\mathrm{BZ}$ and the atomic configuration in a surface unit cell are shown as insets. Large filled, small filled, and small open circles denote $\mathrm{K}$, surface, and subsurface $\mathrm{Si}$ atoms, respectively. The zero of energy is set at $E_{\mathbf{F}}$.

In agreement with the upward shift of the calculated $E_{\mathrm{F}}$, Tochihara et al. ${ }^{5}$ observed that the bulk $\mathrm{Si}-3 p$ and the $\pi$-band photoemission peaks shift downward rapidly at low coverage, but become stable at high coverage. A new peak near $E_{\mathrm{F}}$ was interpreted as arising from the gradual filling of the Cs- $6 s$ band, and thus being due to the metallization of the overlayer. ${ }^{5}$ These observed features are reproduced by us, but obtain a different interpretation in our calculation for the $\mathrm{K}$ overlayer. The appearance of a new peak near $E_{\mathrm{F}}$ can certainly be attributed to the metallization of the overlayer-substrate system. However, here the essential question is whether the substrate or overlayer has metallized. This issue is well resolved for many metal-semiconductor junctions, for example $\mathrm{Al}-\mathrm{Ge}$, where strong and long-range interaction among metal atoms overcomes the Al-Ge chemisorp- 
tion bonds. That was shown to result in the relaxation and weakening of the $\mathrm{Al}-\mathrm{Ge}$ bonds at increased $\mathrm{Al}$ deposition, and eventually the metallization of the overlayer. ${ }^{6}$ As revealed from our charge-density analysis, the present situation with $\mathrm{K}$ is strikingly different.

The charge density of $\pi$ and $\pi^{*}$ states of the clean $\mathrm{Si}$ surface are shown for $\mathbf{k}=0$ in Figs. 2(a) and 2(b), respectively. Note that the bonding charge between two chain atoms occurs below the bond. Clearly, the charge distribution of these states depends upon the wave vector. For example, along the $J K$ direction, the $\pi$ or $\pi^{*}$ state is localized only at one of the surface atoms, with a negligible interatomic coupling, and hence a small superlattice energy gap. The charge distributions of the $\pi$ state for $\Theta=1$ and $\Theta=2$ are practically unaltered. The charge distribution of the $\pi^{*}$ is not affected by $\mathrm{K}$ adsorption so long as this state lies in the gap. When it merges in the conduction band, the charge distribution is slightly polarized because of the nearby $\mathrm{K}$ cores [Figs. 2(c) and $2(d)]$. That the states occupied above the threshold coverage are extended conduction-band states is clarified from an extensive charge-density analysis of states near $E_{\mathrm{F}}$. The comparison of the charge distribution of these states at $\Theta=0,1$, and 2 clearly shows that their bulk conduction-band character being antibonding and spreading over the substrate prevails even at saturation coverage. The effect of the overlayer and the modifications of the upper-lying states are observed by the comparison of the $s$ state of the unsupported $\mathrm{K}$ layer with a surface resonance above $E_{\mathrm{F}}$ in Figs. $2(\mathrm{e})$ and $2(\mathrm{f})$.

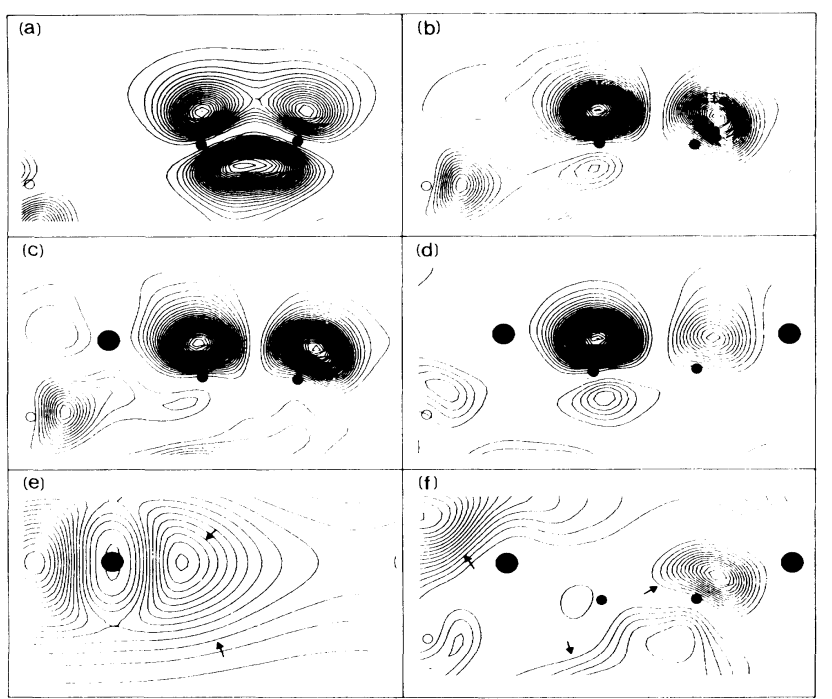

FIG. 2. Charge-density contour plots of the states at $\Gamma$ in a vertical plane passing through a surface $\mathrm{Si}-\mathrm{Si}$ bond: (a) $\pi$ state; (b) $\pi^{*}$ state of the clean $\operatorname{Si}(111)-(2 \times 1)$; (c) $\pi^{*}$ state at $\Theta=1$; (d) $\pi^{*}$ state at $\Theta=2$; (e) $s$ state of the unsupported $\mathrm{K}$ layer; (f) a surface resonance above $E_{\mathrm{F}}$ at $\Theta=2$. The contour spacing is 0.000213 a.u.
The partial filling of the $\pi^{*}$ band and the metallization of the whole substrate are easily seen in Fig. 3. The charge-density contour plots of the clean substrate in the surface plane [Fig. 3(a)] show the chain structure with the bond charge between surface silicons. In a plane above the surface the charge density is lower, but the corrugation due to the chain structure is maintained [Fig. 3(b)]. At $\Theta=1$ and $\Theta=2$, we observe enhancement of charge density over the chain atoms with maxima corresponding to surface silicons [Fig. 3(c)]. Partial filling of the conduction-band states at $\Theta=2$ leads to further modifications in the charge distribution [Fig. 3(d)]. Note that $\mathrm{K}$ atoms at the charge-density hole mimic the zigzag $\pi$ chain. The unsupported $\mathrm{K}$ layer with two $\mathrm{K}$ atoms in the same lattice registry as the overlayer, on the other hand, gives rise to a chain of higher charge density at the opposite side of the surface unit cell. This is where the charge would be found if the overlayer were metallized. A clear picture of the binding and the position of the $\mathrm{K}$ atom relative to the surface charge distribution is illustrated in Fig. 3(e). In this vertically cut plane, two holes of the surface charge density lie at both

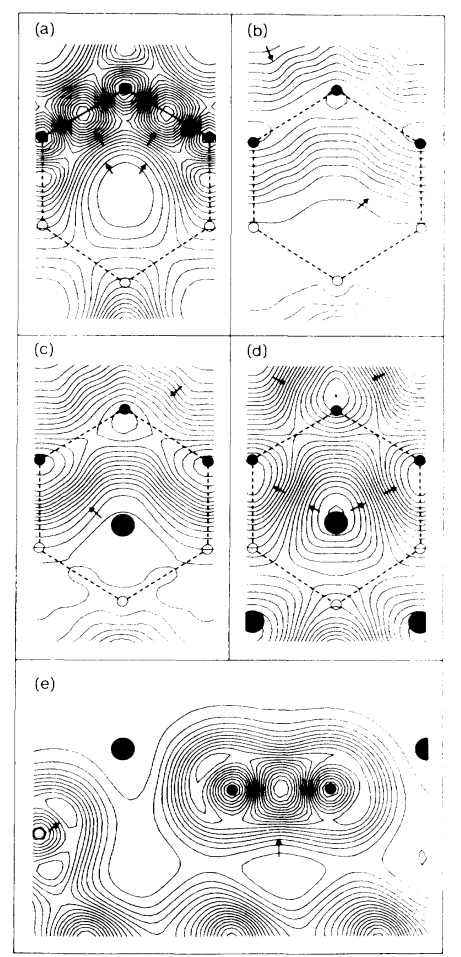

FIG. 3. Contour plots of the total charge density. (a) Charge density of the clean $\mathrm{Si}(111)-(2 \times 1)$ in the surface plane. In a horizontal plane 4 a.u. above the surface, (b) clean surface, (c) one-K-adsorbed surface, (d) two-K-adsorbed surface. In a vertical plane passing through the surface $\mathrm{Si}-\mathrm{Si}$ bond, (e) two-K-adsorbed surface. Contour spacings are 0.005 a.u. for (a), 0.000213 a.u. for (b)-(d), and 0.00448 a.u. for (e). 
sides of the surface $\mathrm{Si}-\mathrm{Si}$ bond, where two $\mathrm{K}$ atoms are located at $\Theta=2$.

From the analysis of the electronic structure and charge density, it becomes clear that even at the saturation coverage the potassium atoms do not form metallic bonds. This prediction contrasts $\mathrm{Si}$ surfaces with metal surfaces, which are known to allow for the metallization of the alkali-metal overlayer at high coverage. ${ }^{7}$ Earlier, ${ }^{8}$ the onset of electron-energy-loss spectroscopy peaks at $\Theta=0.5$ for the $\mathrm{K}$-adsorbed $\mathrm{Si}(001)-(2 \times 1)$ surface were interpreted to imply that the $1 \mathrm{D}$ plasmon excitation of the overlayer was undergoing a Mott transition. However, $\mathrm{Si}(001)-(2 \times 1)$ has an empty band lying totally in the band gap, and calculations ${ }^{9}$ show that the metallic character attributed to the overlayer is, in fact, the metallization of the surface-state band. As noted, here the interaction between $\mathrm{K}$ and $\mathrm{Si}(111)$ is different, and the novel aspect is that beyond a threshold coverage not only the surface but also the silicon substrate undergoes a metallic transition. Recently, Ishida, Terakura, and Tsuka$\mathrm{da}^{10}$ reported a linear combination of atomic orbitals band calculation of the same system. Their conclusions on the metallization are at variance with the present results, perhaps because of the limited basis set and the unoptimized geometry they employed in their study.

In summary, we have investigated the $\mathrm{K}$-adsorbed $\mathrm{Si}(111)$ surface as a function of coverage. We have shown that, because of the existence of the active surface-state band, bonding is strong and ionic. At the initial coverage, electrons donated by $\mathrm{K}$ atoms fill the $\pi^{*}$ band and yield a 1D metallic chain. An important result of this study is that above a threshold coverage, alkalimetal electrons are accommodated by the substrate conduction-band states. This leads to a change in the dimensionality of the metallic system from 1 to 2 (or to 3 in a thick substrate) and results in an enhanced conductivity perpendicular to the surface. Moreover, in a thin $\mathrm{Si}$ substrate with a positively charged surface conduction-band electrons are expected to create a quantum regime similar to one usually obtained in a selectively doped semiconductor superlattice. Finally, since the concentration of itinerant electrons in the conduction band can be controlled, one may envisage creating a degenerate semiconductor without the artifacts of bulk alloying.

${ }^{(a)}$ Permanent address: Department of Physics, Bilkent University, Ankara, Turkey.

(b) Permanent address: Almaden Research Center, Mail Stop K33/801, 650 Harry Road, San Jose, CA 95120.

${ }^{1}$ K. C. Pandey, Phys. Rev. Lett. 47, 1913 (1981).

${ }^{2}$ M. Schlüter, J. R. Chelikowsky, S. G. Louie, and M. L. Cohen, Phys. Rev. B 12, 4200 (1975).

${ }^{3}$ S. Ciraci and I. P. Batra, Phys. Rev. B 33, 4294 (1986).

${ }^{4}$ J. E. Northrup and M. L. Cohen, Phys. Rev. Lett. 49, 1349 (1982).

${ }^{5}$ H. Tochihara, M. Kubota, M. Miyao, and Y. Murata, Surf. Sci. 158, 497 (1985).

${ }^{6}$ I. P. Batra and S. Ciraci, Phys. Rev. B 33, 4312 (1986).

${ }^{7}$ A. U. MacRae, K. Müller, J. J. Lander, J. Morrison, and J. C. Phillips, Phys. Rev. Lett. 22, 1048 (1969).

${ }^{8}$ H. Tochihara, Surf. Sci. 126, 523 (1983); T. Aruga, H. Tochihara, and Y. Murata, Phys. Rev. Lett. 53, 372 (1984).

${ }^{9}$ S. Ciraci and I. P. Batra, Phys. Rev. Lett. 56, 877 (1986).

${ }^{10}$ H. Ishida, K. Terakura, and M. Tsukada, Solid State Commun. 59, 365 (1986). 